\title{
Glycemic Variability Is Independently Associated With Poor Prognosis in Five Pediatric ICU Centers in Southwest China
}

\author{
Milan Dong ${ }^{1,2}$, Wenjun Liu ${ }^{1}$, Yetao Luo ${ }^{3}$, Jing Li ${ }^{1 *}$, Bo Huang ${ }^{4}$, Yingbo Zou ${ }^{4}$, Fuyan Liu ${ }^{4}$, \\ Guoying Zhang ${ }^{5}$, Ju Chen ${ }^{5}$, Jianyu Jiang ${ }^{6}$, Ling Duan ${ }^{6}$, Daoxue Xiong ${ }^{6}$, Hongmin Fu $^{7}$ and \\ Kai $\mathrm{Yu}^{7}$ \\ ${ }^{1}$ Department of Critical Care Medicine, Children's Hospital of Chongqing Medical University, National Clinical Research \\ Center for Child Health and Disorders, Ministry of Education Key Laboratory of Child Development and Disorders, \\ Chongqing, China, ${ }^{2}$ Department of Pediatrics, The People's Hospital of Yubei District of Chongqing City, Chongqing, China, \\ ${ }^{3}$ Department of Clinical Epidemiology and Biostatistics, Children's Institute of Children's Hospital of Chongqing Medical \\ University, Chongqing, China, ${ }^{4}$ Department of Pediatric Critical Care, The First People's Hospital of Zunyi, Zunyi, China, \\ ${ }^{5}$ Department of Pediatric Critical Care, Chengdu Women's and Children's Central Hospital, Chengdu, China, ${ }^{6}$ Department of \\ Pediatrics, Chongqing Three Gorges Women and Children's Hospital, Chongqing, China, ${ }^{7}$ Department of Pediatric Critical \\ Care, Kunming Children's Hospital, Kunming, China
}

OPEN ACCESS

Edited by:

Caterina Conte,

Università telematica San

Raffaele, Italy

Reviewed by:

Yoshifumi Saisho,

Keio University School of

Medicine, Japan

Ariana Raquel Pichardo-Lowden, Penn State Milton S. Hershey Medical

Center, United States

${ }^{*}$ Correspondence:

Jing Li

lijingwangyi@126.com

Specialty section:

This article was submitted to Clinical Nutrition,

a section of the journal

Frontiers in Nutrition

Received: 13 August 2021 Accepted: 17 January 2022

Published: 23 February 2022

Citation:

Dong M, Liu W, Luo Y, Li J, Huang B,

Zou Y, Liu F, Zhang G, Chen J,

Jiang J, Duan L, Xiong D, Fu H and

Yu K (2022) Glycemic Variability Is

Independently Associated With Poor

Prognosis in Five Pediatric ICU

Centers in Southwest China.

Front. Nutr. 9:757982.

doi: 10.3389/fnut.2022.757982
Background: Glucose variability (GV) is a common complication of dysglycemia in critically ill patients. However, there are few studies on the role of GV in the prognosis of pediatric patients, and there is no consensus on the appropriate method for GV measurement. The objective of this study was to determine the "optimal" index of GV in non-diabetic critically ill children in a prospective multicenter cohort observational study. Also, we aimed to confirm the potential association between GV and unfavorable outcomes and whether this association persists after controlling for hypoglycemia or hyperglycemia.

Materials and Methods: Blood glucose values were recorded for the first $72 \mathrm{~h}$ and were used to calculate the GV for each participant. Four different metrics [SD, glycemic lability index (GLI), mean absolute glucose (MAG), and absolute change of percentage (ACACP)] were considered and compared to identify the "best" GV index associated with poor prognosis in non-diabetic critically ill children. Among the four metrics, the SD was most commonly used in previous studies, while GLI- and MAG-integrated temporal information, that is the rate and magnitude of change and the time interval between glucose measurements. The fourth metric, the average consecutive ACACP, was introduced in our study, which can be used in real-time clinical decisions. The primary outcome of this study was the 28-day mortality. The receiver operating characteristic (ROC) curve analysis was conducted to compare the predictive power of different metrics of GV for the primary outcome. The GV index with the largest area under ROC curve (AUC) was chosen for subsequent multivariate analyses. Multivariate Cox regression analysis was performed to identify the potential predictors of the outcome. To compare the contribution in 28-day mortality prognosis between glycemic variability and hyper- or hypoglycemia, performance metrics were calculated, which included AUC, net reclassification improvement (NRI), and integrated discrimination improvement (IDI). 
Results: Among 780 participants, $12.4 \%(n=97)$ died within 28 days after admission to the pediatric intensive care unit (PICU). Statistically significant differences were found between survivors and non-survivors in terms of four GV metrics (SD, GLI, MAG, and ACACP), in which MAG (AUC: 0.762, 95\% Cl: 0.705-0.819, $p<0.001$ ) achieved the largest $A \cup C$ and showed a strong independent association with ICU mortality. Subsequent addition of MAG to the multivariate Cox model for hyperglycemia resulted in further quantitative evolution of the model statistics ( $A \cup C=0.651-0.681, p=0.001$; IDI: $0.017, p=0.044 ; N R I: 0.224, p=0.186$ ). The impact of hyperglycemia (adjusted hazard ratio [aHR]: $1.419,95 \% \mathrm{Cl}: 0.815-2.471, p=0.216$ ) on outcome was attenuated and no longer statistically relevant after adjustment for MAG (aHR: 2.455, 95\% Cl: 1.411-4.270, $p=0.001$ ).

Conclusions: GV is strongly associated with poor prognosis independent of mean glucose level, demonstrating more predictive power compared with hypoglycemia and hyperglycemia after adjusting for confounding factors. GV metrics that contain information, such as time and rate of change, are the focus of future research; thus, the MAG may be a good choice. The findings of this study emphasize the crucial role of GVs in children in the PICU. Clinicians should pay more attention to GV for clinical glucose management.

\section{Keywords: dysglycemia, glycemic variability, MAG, pediatrics, critically ill, mortality}

\section{INTRODUCTION}

Glucose management is an important element of intensive care management. In general, glucose dysregulation is related to negative outcomes in critically ill patients and is a multi-system complication (1). A few authors have explored the effectiveness of glucose levels on the prognosis of glucose dysregulation with different glucose management protocols; however, the results are contradictory, especially regarding the question of whether strict control of glucose levels is linked to improve clinical prognosis $(2,3)$. Increased glucose variability $(\mathrm{GV})$ increases the risk of poor outcomes, which may explain the contradictory results in the literature (4).

In 2006, Egi et al. (5) demonstrated for the first time that GV is closely related to poor outcomes in critically ill adults. In recent years, a growing number of studies have confirmed that GV is independently associated with unfavorable outcomes in critically ill adults, regardless of its definition (6-10). However, fewer studies, which explored the impact of GV on negative prognosis have focused on pediatric populations, compared to adults,

Abbreviations: ACACP, Average consecutive absolute change percentage; Adm, Blood glucose concentration at admission; AUC, Area under the curve; BAZ, Body mass index for age Z score; BMI, Body mass index; CPR, Cardiopulmonary resuscitation; CRRT, Continuous renal replacement therapy; GIR, Glucose infusion rate; GLI, Glycemic lability index; HR, Hazard Ratio; MAG, Mean absolute glucose; Mean, Average blood glucose; MODS, Multi-organ dysfunction syndrome; MV, Mechanical ventilation; PICU, Pediatric intensive care unit; ROC, The receiver operating characteristic; TGC, Tight glycemic control; PIM2, Pediatric index of mortality score II; +LR, Positive likelihood ratio; -LR, Negative likelihood ratio; $+\mathrm{PV}$, Positive predictive value; $-\mathrm{PV}$, Negative predictive value; IQR, Interquartile range; ROS, Reactive oxygen species; NFאB/RAGE, The nuclear factor-kappa B/Receptor for advanced glycation end products. and most of the previous studies $(11,12)$ were retrospectively conducted in a single center. Thus, prospective multicenter studies are needed on the association between adverse prognosis and GV in pediatric patients. Subsequently, we conducted this study with a prospective analysis of a pediatric cohort to address this clinical issue.

The GV, also known as glucose fluctuation, represents the fluctuation of glucose levels over time and refers to a nonstationary state in which glucose levels fluctuate between high and low values, such as speed, magnitude, and frequency. A few GV metrics appear to be correlated with worse outcomes in critically ill patients, such as standard deviation $(7,8,13)$, coefficient of variation $(7,13,14)$, mean amplitude of glycemic excursion $(7,13,15)$, mean absolute glucose $(13,16)$, and glycemic lability index (GLI) (15). These measurements differ in the requirements for calculations, such as the frequency or time interval between glucose measurements. Nevertheless, there is currently no consensus on the definition of GV and the "best" index.

Our retrospective study (17) also demonstrated that GV was independently associated with unfavorable outcomes. In addition to $\mathrm{GV}$, hyperglycemia and hypoglycemia have been previously reported to be associated with poor prognosis in pediatric patients $(11,18-20)$. Therefore, in this study, we prospectively investigated whether the correlation between GV and poor prognosis remained after controlling for hyperglycemia and hypoglycemia. This study aimed to broaden the understanding of the ways in which GV, hypoglycemia, and hyperglycemia are associated with adverse clinical outcomes in children admitted to the pediatric intensive care units (PICUs) and to improve our understanding of glucose management. 


\section{MATERIALS AND METHODS}

\section{Study Design and Quality Control}

This was a prospective, multicenter, observational study conducted between January 1, 2020 and December 31, 2020. The participants were recruited from five PICUs that belong to tertiary A hospitals in southwest China and represent the top intensive care centers, providing top-tier medical care to most critically ill children in these regions. The ethical committee of the Children's Hospital of Chongqing Medical University approved this study (File No. 2019,37), and the clinical trial registration was completed (ChiCTR2000030846).

Based on the literature, a sample size of 665 can produce $90 \%$ power at the $5 \%$ level of significance when considering a $20 \%$ lost-to-review rate, which was calculated based on the primary outcome-28-day mortality in PICU with cohort study analysis using an estimated intensive care unit (ICU) mortality of $10 \%$ and a risk ratio of $2(21,22)$.

Before initiating the study, we provided professional training for data loggers in each research PICU, including project objectives, research progress, and data collection. Multi-center data collection was performed by recorders under strict guidelines. After data collection and entry, the data were sent to a central repository where they were corrected for any suspicious errors or missing values by the chief coordinator and physicians.

\section{Inclusion and Exclusion Criteria}

All critically ill children aged 36 weeks (corrected gestational age) to 16 years who were receiving vasoactive drug support for hypotension or ventilatory support for respiratory failure was considered for inclusion. Exclusion criteria included the following children: (1) those who remained in the PICU for $<24 \mathrm{~h}$, (2) those who were diagnosed with diabetes mellitus or inborn errors of metabolism, (3) those who had utilized glucocorticoids or total parenteral nutrition (TPN) treatment for 3 days after PICU admission; (4) those who were readmitted to the PICU.

\section{Data Collection}

Data were manually entered into the Epidata software, version 3.1. For each patient, demographic data (age, sex, and nutritional status), clinical treatments [vasoactive and glucose infusions, mechanical ventilation (MV), and other adjuvant therapies], and prognosis (28-day mortality, multi-organ dysfunction syndrome (MODS), and length of time in the PICU) were recorded. The primary outcome was 28-day mortality. Secondary outcomes were the number of days alive and free from MV at 28 days (ICU free days to day 28, MV free days to day 28) after admission. If the patient died within 28 days, we recorded a 0 . If a patient survived for a period within 28 days, we recorded 28 minus the survival time; the smaller this value, the worse the prognosis, which is similar to the record of MV free days to day 28. In addition, the incidence of MODS was recorded as a poor prognosis indicator. The follow-up for each patient admitted to our PICUs was performed and recorded by the nurse 1 month after discharge using telephone interviews (the questionnaire is shown in Supplementary Material). We chose the Pediatric
Index of Mortality-2 (PIM2) score (23) to estimate the severity of the illness at the time of admission to the PICUs. The index was used to determine nutritional status as the " $z$ " score for weight/height ( $\leq 5$ years old) or BMI ( $>5$ years old) for age using the Epi-Info software. Overweight was defined as BMI for age $>+2 \mathrm{SD}$, and wasting was $<-2 \mathrm{SD}$.

\section{Blood Glucose Indices}

We collected all blood glucose values for the first $72 \mathrm{~h}$ after admission. The children in the PICU were examined for blood gases at least twice per day based on their daily treatment. Consequently, no additional blood specimens were performed. The specimens were mainly from arterial blood but also included venous and capillary blood sources.

For each patient, we calculated the following metrics (the reasons for GV metrics included in our study are shown in Supplementary Material) based on all the available glucose values: the average glucose (Mean), SD, GLI [GLI $\left.\left.=\sum\left(\Delta \mathrm{BG}^{2} / \Delta \mathrm{h}\right)\right] / \mathrm{d},(\mathrm{mmol} / \mathrm{L}) 2 / \mathrm{h} / \mathrm{d}\right)(7)$, and mean absolute glucose $(\mathrm{MAG})\left[\mathrm{MAG}=\left(\sum \Delta \mathrm{BG}\right) / \Delta \mathrm{h},(\mathrm{mmol} / \mathrm{l})\right]$ (16). In addition, the average consecutive absolute change percentage $\left[\mathrm{ACACP}=1 /(\mathrm{ni}-1) * \sum(|\mathrm{Xi}-\mathrm{Xi}-1| / \mathrm{Xi}), \%\right]$ (24) was introduced. Indeed, an index can be conveniently used in realtime to aid with clinical decisions. Glucose measurement upon admission (Adm) was the first glucose value after ICU admission. We chose to define hypoglycemia as a glucose value $\leq 3.6 \mathrm{mmol} / \mathrm{L}$ and hyperglycemia cut-off values of $8.3 \mathrm{mmol} / \mathrm{L}$ based on both adult and pediatric studies $(11,25)$.

\section{Blood Glucose Management}

As per the existing unit policy, dextrose infusion is stopped when the glucose value exceeds $15 \mathrm{mmol} / \mathrm{L}$ and actively deals with the primary disease (26). Then, we observe for $1 \mathrm{~h}$ : if there was no downward trend (glucose decreased to $<4 \mathrm{mmol} / \mathrm{L} / \mathrm{h}$ ), we could infuse insulin $(0.05-0.1 \mathrm{U} / \mathrm{kg})$. Insulin infusion was stopped when the blood glucose level was below $10 \mathrm{mmol} / \mathrm{L}(27,28)$.

\section{Statistical Analysis}

Statistical analyses were performed using the $\mathrm{R}$ version 4.0.0. Categorical variables were described by $n$ (\%), and continuous variables without normal distribution were described by the median (IQR). For comparisons between the two groups, the Wilcoxon rank-sum test was used for continuous variables without normal distribution, while the chi-square test was used for categorical variables. We used ROC curve analysis to compare the predictive power of different indicators of GV for the primary outcome. The GV index with the largest area under ROC curve (AUC) was also converted to a categorical variable based on the cut-off value for subsequent analysis. The GV index and hypo/hyperglycemia were gradually substituted into a multivariate Cox regression model adjusted for confounders with a significance level of $p \leq 0.10$ to prevent the exclusion of significant variables from the regression model. The results are expressed as hazard ratio (HR) $(95 \%$ CI). The overall predictive accuracy of the model was quantified using the AUC. In addition, net reclassification improvement (NRI) and integrated discrimination improvement (IDI) analyses (29) 


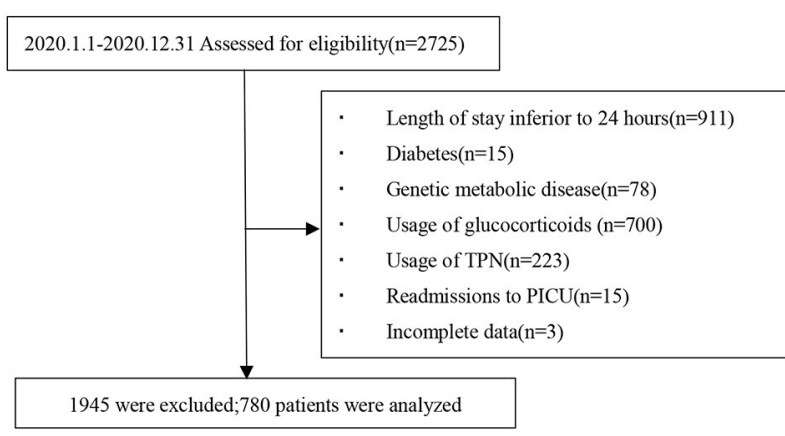

FIGURE 1 | Flowchart illustrating the inclusion and exclusion of participants for the study.

were performed to assess GV and the predictive power of hypoglycemia/hyperglycemia. Depending on the type of outcome variable, multivariable logistic regression or linear regression can also be used to assess the impact of GV on other outcome indicators (e.g., ICU-free days or ventilator-free days to day 28, and MODs). A value of $p<0.05$ for a two-sided test is considered statistically significant.

\section{RESULTS}

We consecutively monitored a total of 2,725 patients in the five participating units during the study period in which 780 patients met the nadir criteria for entry into the study. A flowchart of the case screening is shown in Figure 1.

In total, $97(12.4 \%)$ of the 780 participants died within the 28-day period after PICU admission. At the primary endpoint (28-day mortality), participants were divided into survival and non-survival groups. The primary reasons for ICU admission included 353 cases of respiratory diseases (such as severe pneumonia and asthma), 35 cases of cardiovascular diseases (such asshock), 130 cases of neurological diseases, 85 cases of trauma, 14 cases of postoperative care, 58 cases of sepsis, and 105 other cases (such as oncologic, renal, metabolic, and hematologic cases). A total of 9,142 glucose values were collected of which $65.9 \%$ were from arterial blood gas analysis, $3.4 \%$ from venous blood, and $30.7 \%$ from capillary blood, with a median frequency of 11 (IQR: 8.25-14) glucose monitoring per patient. Demographic information, clinical features, and disease severity between the survival and non-survival groups are shown in Table 1.

Table 2 illustrates the comparative differences in glucose metrics between the survival and non-survival groups, showing that all glucose metrics were significantly lower in the survivors group than in the non-survivors group $(p<0.05)$.

The AUCs are shown in Table 3, which were plotted to evaluate the ability of glucose metrics and PIM2 score in predicting 28-day PICU mortality. These results separately correlated with PICU 28-day mortality $(A U C>0.700, p<$ 0.05). However, MAG (AUC: 0.762, 95\% CI: 0.705-0.819, $p<$ 0.001 ) has the largest areas compared to other GV indices in the prediction of PICU 28-day mortality. MAG had significantly better discrimination of PICU 28-day mortality than the other metrics $(p<0.05)$. Therefore, we used MAG to represent GV and explore its impact on adverse outcomes. Additionally, we utilized an optimal cut-off value for 28 -day mortality prediction.

We sequentially included the baseline variables in the Cox regression model with $p \leq 0.1$ in Table 1 , followed by hypoglycemia and MAG, respectively. The results of the multivariate regression model showed that hypoglycemia was not associated with 28-day mortality (Table 4, Model 2) after adjusting for significant confounders. However, GV represented by MAG (adjusted HR: 2.795, 95\% CI: 1.6874.632, $p<0.001$ ) was still related to PICU mortality after adjustment for hypoglycemia (Table 4, Model 3). The addition of MAG to the model for hypoglycemia led to further quantitative evolution of the model statistics (AUC: from 0.616 to $0.668, p<0.001$; IDI: 0.030, $p=0.006$; NRI: $0.325, p=0.022$ ). There was no interaction between MAG and hypoglycemia.

We constructed the same model described above by replacing hypoglycemia with hyperglycemia. After adjustment for confounding factors, patients with hyperglycemia experienced increased rates of 28-day mortality (adjusted $H R$ for hyperglycemia, $2.026 ; 95 \% C I: 1.240-3.310 ; p=0.005$ ) (Table 5, Model 2). Subsequent addition of MAG to the model for hyperglycemia resulted in an early incremental improvement in model statistics (AUC: from 0.651 to $0.681, p=0.001$; IDI: $0.017, p=0.044 ; N R I: 0.224, p=0.186)$. After adjusting for MAG (adjusted HR: 2.455, 95\% CI: 1.411-4.270, $p=0.001$ ), the effect of hyperglycemia (adjusted HR: 1.419, 95\% CI: 0.815-2.471, $p=$ 0.216) (Table 5, Model 3) on the adverse outcome was attenuated and no longer statistically relevant. In the final model, MAG had a greater prognostic contribution than hyperglycemia $\left(\chi^{2}-d f\right.$ (30): 9.115 and 0.528 for MAG and hyperglycemia, respectively).

However, the interaction between hyperglycemia and MAG was statistically significant $(p=0.016)$. We used Cochran's and Mantel-Haenszel statistics to further explore the relationship between hyperglycemia, MAG, and mortality (Figure 2). Higher mortality was observed in the high GV group than in the low GV group ( $p<0.001$ ), regardless of the presence of hyperglycemia. The test of homogeneity of the odds ratio (OR) was $p=$ 0.022; therefore, after stratifying by MAG, in the low GV group, hyperglycemia was relevant to PICU mortality $(O R=2.910,95 \%$ $C I: 1.513-5.596, p=0.001)$. In the high GV group, hyperglycemia did not affect mortality $(O R=0.846$; 95\% CI: $0.363-1.974, p=$ 0.699). This means that patients with hyperglycemia will have different mortality rates depending on the MAG. In contrast, in the high GV group, hyperglycemia did not play a role in mortality events. These results support the possibility that GV may be a stronger predictor of 28-day mortality than hyperglycemia.

Table 6 shows the effect of blood fluctuations on ICU-free days to day 28, MVfree days to day 28, and the incidence of MODSs. The results show that the median ICU-free days in the high variability group were lower than that of the low group (15 [0.0-22.9] vs. $22[15.1-24.5], p=0.001)$. Similar results were observed in the ventilator-free days (21.5 [0.0-26.1] vs. 25.7 [21.0-28.0], $p<0.001)$. The incidence of MODs was significantly 
TABLE 1 | The comparison of baseline variables between survivors and non-survivors[M (P25, P75), $n(\%)]$.

\begin{tabular}{|c|c|c|c|c|c|}
\hline & All $(n=780)$ & Survivors $(n=683$ ) & Non-survivors $(n=97)$ & $x^{2} / Z$ & $P$ value \\
\hline \multicolumn{6}{|l|}{ Age group/n (\%) } \\
\hline$<3 y s$ & 542.(69.5) & 483.(70.7) & 59.(60.8) & & \\
\hline 3ys to <16ys & 238 (30.5) & $200(29.3)$ & $38(39.2)$ & 3.920 & 0.059 \\
\hline Male/n (\%) & $464(59.5)$ & $404(59.2)$ & $60(61.9)$ & 0.258 & 0.659 \\
\hline Medical/n (\%) & 600 (76.9) & $522(76.4)$ & 78 (80.4) & 0.760 & 0.441 \\
\hline$-2 \sim 2$ & 551 (70.6) & $481(70.4)$ & $70(72.2)$ & & \\
\hline$>2$ & $56(7.2)$ & $53(7.8)$ & $3(3.1)$ & 2.941 & 0.232 \\
\hline $\mathrm{MV} / \mathrm{n}(\%)$ & 588 (75.4) & 495 (72.5) & $93(95.9)$ & 25.068 & $<0.001$ \\
\hline CRRT/n (\%) & $51(6.5)$ & $29(4.2)$ & $22(22.7)$ & 47.233 & $<0.001$ \\
\hline CPR/n (\%) & $26(3.3)$ & $11(1.6)$ & 15 (15.5) & - & $<0.001$ \\
\hline PIM2 score & $-2.6(-3.3,-1.9)$ & $-2.6(-3.5,-2.1)$ & $-1.4(-2.6,1.9)$ & -7.240 & $<0.001$ \\
\hline
\end{tabular}

TABLE 2 | The comparison of glucose metrics between survivors and non-survivors [M (P25, P75), $n(\%)]$.

\begin{tabular}{|c|c|c|c|c|c|}
\hline Metrics & All $(n=780)$ & Survivors $(n=683)$ & Non-survivors $(n=97)$ & $c^{2} / Z$ & $P$ value \\
\hline Adm & $5.9(5.1,7.2)$ & $5.8(5.0,7.1)$ & $6.6(5.2,9.6)$ & -3.094 & 0.002 \\
\hline Mean & $5.8(5.4,6.4)$ & $5.8(5.4,6.3)$ & $6.2(5.3,7.9)$ & -2.878 & 0.004 \\
\hline Hyperglycemia/n (\%) & $289(37.1)$ & $224(32.8)$ & $65(67.0)$ & 42.630 & $<0.001$ \\
\hline Hypoglycemia/n (\%) & $155(19.9)$ & $119(17.4)$ & 36 (37.1) & 20.681 & $<0.001$ \\
\hline SD & $1.0(0.7,1.5)$ & $0.9(0.7,1.4)$ & $1.6(1.1,3.0)$ & -7.279 & $<0.001$ \\
\hline ACACP (\%) & $16.9(12.8,23.0)$ & $16.5(12.5,22.2)$ & $22.3(16.4,32.6)$ & -5.788 & $<0.001$ \\
\hline
\end{tabular}

higher in the high-GV group than in the low-GV group (62.9 vs. $29.5 \%, p<0.001)$.

\section{DISCUSSION}

To our knowledge, this is the first multicenter prospective study of pediatric dysglycemia (such as hypoglycemia, hyperglycemia, and GV) conducted in Southwest China, which attempted to establish an association between dysglycemia and 28day mortality in PICUs. Several important relationships were also observed. First, GV was independently associated with unfavorable outcomes, and it may play a more predictive role in PICU mortality than hyperglycemia and hypoglycemia. These findings emphasize the important role of GV in children admitted to the PICU. Also, MAG may be a dominant index reflecting glucose fluctuations, which considers the speed and magnitude of change, and the time series among glucose values.

A strong relationship between increasing $\mathrm{GV}$ and poor prognosis has been consistently reported in adult patients (610, 31). Few studies in this field have been conducted in pediatrics; however, similar insights have been obtained. In 2006, Wintergerst et al. (11) retrospectively observed that increased
GV was related to increased hospitalization stay and mortality rates in 1,094 PICU admissions in a single center; however, the severity of the disease was ignored. Hirshberg et al. (22) performed a retrospective study of 863 non-diabetic children in the PICU during a 1-year period and found that the incidence of glucose fluctuations (defined as having both hyperglycemia and hypoglycemia) was $6.8 \%$, and glucose fluctuations were still associated with high mortality after adjusting for PRISM score. In another retrospective cohort of 101 critically ill children, researchers found that increased GV was associated with increased mortality (12). Similarly, Bhutia et al. (21) prospectively observed 170 critically ill children and found that GV was related to multi-organ dysfunction. Pinchefsky et al. (32) found that GV was independently associated with the deterioration of brain function in neonates with encephalopathy. Although these studies involved children in a single clinical setting, they add to the evidence that GV is associated with adverse outcomes. In our series, we further prospectively investigated the relationship between GV and deleterious effects in multiple centers, and similar results were reported in our findings. Based on our results, GV was associated with mortality, MODs, and ventilator/ICUfree days, which confirmed the strong predictive role of $\mathrm{GV}$ 
TABLE 3 | Receiver operating characteristic curves for mortality on critically ill children.

\begin{tabular}{|c|c|c|c|c|c|c|c|}
\hline & AUC $(95 \% \mathrm{Cl})$ & $\begin{array}{c}\text { Cutoff value } \\
\text { (sensitivity/specificity, } \\
\% \text { ) }\end{array}$ & $\boldsymbol{P}$ 值 & +LR & -LR & $+\mathrm{PV}$ & $-P V$ \\
\hline Adm & $0.597(0.530,0.664)$ & $6.70(48.45,71.89)$ & 0.002 & 1.72 & 0.72 & 19.7 & 90.8 \\
\hline PIM2 & $0.727(0.668,0.786)$ & $-1.88(56.70,81.55)$ & $<0.001$ & 3.07 & 0.53 & 30.4 & 93.0 \\
\hline Mean & $0.590(0.518,0.662)$ & $7.03(32.99,90.48)$ & 0.004 & 3.47 & 0.74 & 33.0 & 90.5 \\
\hline SD & $0.728(0.671,0.785)$ & $1.11(75.26,62.23)$ & $<0.001$ & 1.99 & 0.40 & 22.1 & 94.7 \\
\hline GLI & $0.729(0.670,0.789)$ & $4.37(57.73,79.65)$ & $<0.001$ & 2.84 & 0.53 & 28.7 & 93.0 \\
\hline MAG & $0.762(0.705,0.819)$ & $0.27(59.79,82.58)$ & $<0.001$ & 3.43 & 0.49 & 32.8 & 93.5 \\
\hline ACACP & $0.681(0.621,0.742)$ & $24.20(47.42,81.55)$ & $<0.001$ & 2.57 & 0.64 & 26.7 & 91.6 \\
\hline
\end{tabular}

TABLE 4 | Multivariable COX regression: associations of hypoglycemia and MAG With 28-day mortality.

\begin{tabular}{|c|c|c|c|c|c|c|}
\hline Sequential models for 28 -day mortality & Main variables & HR (95\%Cl) & $P$ value & \multicolumn{3}{|c|}{ Model statistics } \\
\hline Multivariable model 1: & Mean & $1.049(0.928,1.187)$ & 0.443 & 290.04 & - & $0.607(0.541,0.674)$ \\
\hline \multirow[t]{2}{*}{ Multivariable model 2: model1+hypoglycemia } & Mean & $1.065(0.942,1.205)$ & 0.313 & 296.739 & 0.168 & $0.616(0.552,0.681)$ \\
\hline & Hypoglycemia & $1.415(0.870,2.300)$ & 0.162 & & & \\
\hline & MAG & $2.795(1.687,4.632)$ & $<0.001$ & & & \\
\hline
\end{tabular}

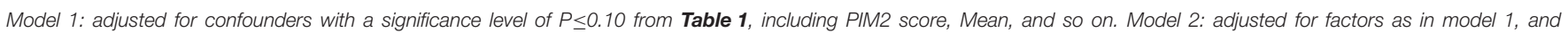
hypoglycemia. Model 3: adjusted for factors as in model 2, and MAG.

${ }^{\ddagger}$ For likelihood ratio test between sequential models; the P-value is for comparison of the multivariable model to the previous model.

in children in the PICU. This association may be attributed to oscillatory glucose induction that exacerbates oxidative stress, promotes inflammatory responses, and regulates ROS-mediated NFKB/RAGE activation, resulting in endothelial cell injury (3335). Taken together, these results emphasize the dangerous role of $\mathrm{GV}$ in critically ill patients. Indeed, management of GV may be warranted in glucose management regimens.

Our data are also complementary to findings from observational studies that evaluated the association between hypo/hyperglycemia and GV during critical illness and mortality. The results of our study suggest that GV may be a more powerful independent risk factor of mortality in patients in the PICU than hyperglycemia and hypoglycemia. Consistent with our findings, Bagshaw et al. (36) found that glucose fluctuation, defined as having both hypoglycemia and hyperglycemia, was relevant to a larger $O R$ of ICU (1.5 vs. 1.2 vs. $1.0, p<0.05)$ and hospital ( 1.4 vs. 1.2 vs. $1.0, p<0.05)$ mortality, compared to only hypoglycemia or hyperglycemia, or neither. Several in vitro or animal experiments (37-39) have demonstrated that oscillating glucose levels aggravate oxidative stress compared to persistent hyperglycemia, resulting in accelerated cell injury and apoptosis. Quagliaro et al. (37) demonstrated that in umbilical vein cells, the levels of protein kinase C- $\beta$ were higher in the rapid glucose fluctuation group than in the sustained hyperglycemia group.

From the perspective of clinical treatment, a single blood glucose value can be influenced by various factors, such as medication, diet, and stress status. Therefore, hyperglycemia or hypoglycemia determined by a single value may not accurately reflect the metabolic status of critically ill patients. In contrast, $\mathrm{GV}$ is calculated using a few glucose values and may reveal dynamic glucose changes. This may be one explanation that glucose fluctuation may be a stronger predictor of mortality than hyperglycemia and hypoglycemia. Therefore, GV should be considered as an important predictor in future studies of prognostic models in critically ill patients, and clinicians should pay more attention to dynamic fluctuations of glucose versus single glucose values.

In contrast to the previous study, which only used a single index that defined GV, our study selected four indicators (SD, GLI, MAG, and ACACP). ROC analysis showed that three of the four GV indicators (SD, GLI, and MAG) had a moderate predicted value for 28-day PICU mortality (AUC > 0.7, $p<0.05$ ). Among all four indices, MAG obtained the largest AUC and was found to be a better indicator of GV than SD. Hermanides et al. (16) illustrated that the glucose profile demonstrated different MAGs even in the presence of the same SD value, and MAG was superior to SD in terms of predicting mortality. Variability should be considered in terms of the speed and magnitude of change, as well as the time interval between glucose measurements; meanwhile MAG does account for this variability, but $S D$ does not. Our retrospective study (17) found that GLI, a variability indicator that also accounts for the arrangement of estimations 
TABLE 5 | Multivariable COX regression: associations of hyperglycemia and MAG With 28-day mortality.

\begin{tabular}{|c|c|c|c|c|c|c|}
\hline \multirow[t]{2}{*}{ Sequential models for 28 -day mortality } & \multirow[t]{2}{*}{ Main variables } & \multirow[t]{2}{*}{ HR (95\%Cl) } & \multirow[t]{2}{*}{$P$ value } & \multicolumn{3}{|c|}{ Model statistics } \\
\hline & & & & $\begin{array}{l}\text { Likelihood } \\
\text { ratio } \\
\text { chi-square }\end{array}$ & $P^{\ddagger}$ value & AUC \\
\hline Multivariable model 1: & Mean & $1.049(0.928,1.187)$ & 0.443 & 290.04 & - & $0.607(0.541,0.674)$ \\
\hline \multirow[t]{2}{*}{ Multivariable model 2: Model 1+Hyperglycemia } & Mean & $0.980(0.860,1.117)$ & 0.765 & 290.155 & 0.005 & $0.651(0.590,0.712)$ \\
\hline & hyperglycemia & $2.026(1.240,3.310)$ & 0.005 & & & \\
\hline \multirow[t]{3}{*}{ Multivariable model 3: Model 2+MAG } & Mean & $0.942(0.824,1.075)$ & 0.385 & 303.479 & 0.001 & $0.681(0.622,0.739)$ \\
\hline & Hyperglycemia & $1.419(0.815,2.471)$ & 0.216 & & & \\
\hline & MAG & $2.455(1.411,4.270)$ & 0.001 & & & \\
\hline
\end{tabular}

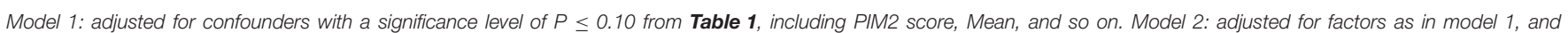
hyperglycemia. Model 3: adjusted for factors as in model 2, and MAG.

${ }_{\ddagger}$ For likelihood ratio test between sequential models; the P-value is for comparison of the multivariable model to the previous model.

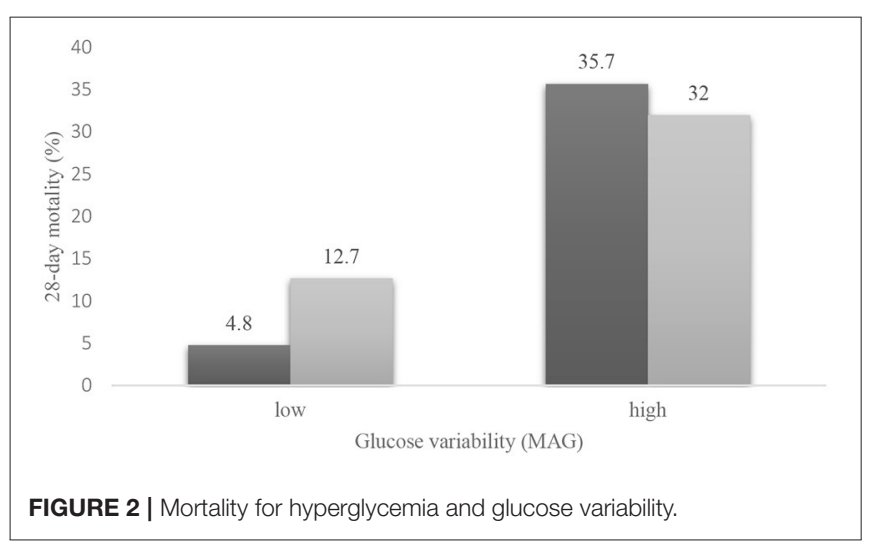

and time, had increased predictive power for PICU mortality compared to SD, CV, and MAGE (AUC: 0.626 vs. $0.601,0.599$, $0.573)$. Based on the results of previous studies $(7,15)$ and our own results, GV index which accounts for the range, speed, time series, should be used; MAG, or GLI may be a good choice. Given its clinical practicality, we introduced ACACP as a GV indicator, which is simple to apply to a treatment protocol (24). This index focused on the comparison of changes in subsequent blood glucose values. Although the AUC of ACACP was smaller than that of MAG in our dataset, its unique calculation and clinical significance suggest that GV metrics should reflect the change from one value to the next and guide clinical management to minimize this change, thereby facilitating glycemic management and improving prognosis in clinical practice. Finding indicators that contain information, such as time series, rate, and range of change, and that are convenient for clinical application is a potential practical target for future treatment.

The American College of Critical Care Medicine suggested that a target BG that is in the range of $100-180 \mathrm{mg} / \mathrm{dL}$ may be a reasonable goal for critically ill pediatric patients (40). In Surviving Sepsis Campaign international guidelines, there is a consensus to target glucose levels below $180 \mathrm{mg} / \mathrm{dL}$ (10 $\mathrm{mmol} / \mathrm{L}$ ) for children with septic shock (41). These guidelines focus only on the level of absolute glucose values. The Clinical
TABLE 6 | Effect of Glucose variability on 28-day ICU-free days, 28-day ventilator-free days, and multiorgan dysfunction syndrome $[\mathrm{M}(\mathrm{IQR}) / n(\%)]$.

\begin{tabular}{|c|c|c|c|}
\hline & \multicolumn{2}{|c|}{ MAG (mmol/L) } & \multirow[t]{2}{*}{$P$ value } \\
\hline & $<0.27$ & $\geq 0.27$ & \\
\hline 28-day ICU-free days & $22.0(15.1,24.5)$ & $15(0.0,22.9)$ & 0.001 \\
\hline 28-day ventilator-free days & $25.7(21.0,28.0)$ & $21.5(0.0,26.1)$ & $<0.001$ \\
\hline Multiorgan dysfunction syndrome & $176(29.1)$ & $110(62.9)$ & $<0.001$ \\
\hline
\end{tabular}

Adjusted for factors with a significance level of $P \leq 0.10$ from Table 1.

Practice Guideline (40) states that GV needs to be used as an indicator of clinical glucose control management. Our findings also suggest that glucose management should not only consider the glucose value, but also GV. The available literature was inadequate to support recommendations regarding GV measurements and standard indices in pediatric patients. The glucose regimen and monitoring system should be designed to minimize glycemic variability in clinical practice. Tracking and minimizing differences from one blood glucose measurement to the next is a potential practical target for future treatment. ACACP as a practical indicator, combined with continuous glucose monitoring (CGM), will likely increase the safety and acceptance of GV management protocols. CGM may be a good choice for practical, real-time treatment goals, regardless of its high price and requirements for use.

The multicenter, prospective design is a major strength of this study, particularly the choice to assess four GV indices in a large cohort of unselected children in the PICU and to explore their relationship with adverse outcomes. The results have a wider applicability than those of single-center studies. In addition, GV in the early stages may reflect a physiological stress response; indeed, as treatment duration increases, it may be influenced by many factors related to treatment, such as nutritional intake and drug usage (42). In the present study, GV metric calculations included the first $72 \mathrm{~h}$ of glucose values and excluded children using glucocorticoids or TPN, reducing the impact of medical management. Thus, GV in our study may predict mortality. 
However, our data on glucose included only the first $72 \mathrm{~h}$ after admission to the PICU, which represents a short period; thus, there may be a bias in the results. However, our large cohort may have eliminated this effect to some extent.

Our study has some limitations. First, there may be ascertainment bias, as patients with abnormal blood glucose levels may have more frequent measurements, while those with normal blood glucose levels have a comparatively reduced number of measurements. As a result, we may miss the record of hyperglycemia or hypoglycemia in some patients. Ideally, CGM should be performed at predetermined time points; however, this will increase the amount of blood sampled and, thereby, the level of discomfort experienced by young children. Advances in CGM technology will facilitate the management or monitoring of early GV in critically ill children. In addition, our glucose measurements were obtained from different blood specimens, and homogeneity could not be ensured. Finally, our study cannot draw conclusions about causality, since the results of observational studies can only assume that an association exists.

\section{CONCLUSIONS}

Our results endorse and expand on previous literature findings by showing that fluctuations in blood glucose control negatively affect clinical prognosis in children in the PICU, and GV may be more strongly associated with mortality outcomes compared to hypoglycemia and hyperglycemia. Clinicians in PICUs should pay more attention to GV. MAG may be the dominant indicator of GV, which contains information on the speed and magnitude of blood glucose changes. These results emphasize the importance of monitoring GVs in children in the PICU, such as the use of the CGM system. Exploring the mechanisms of GV and optimizing personal glycemic management in PICUs will be the focus of future research to minimize the potential risk of healthcare-acquired events.

\section{DATA AVAILABILITY STATEMENT}

The original contributions presented in the study are included in the article/Supplementary Material,

\section{REFERENCES}

1. Chi A, Lissauer ME, Kirchoffner J, Scalea TM, Johnson SB: effect of glycemic state on hospital mortality in critically ill surgical patients. Am Surg. (2011) 77:1483-89. doi: 10.1177/000313481107701138

2. Vlasselaers D, Milants I, Desmet L, Wouters PJ, Vanhorebeek I, Van den $\mathrm{HI}$, et al. Intensive insulin therapy for patients in paediatric intensive care: a prospective, randomised controlled study. Lancet. (2009) 373:54756. doi: 10.1016/S0140-6736(09)60044-1

3. Agus MS, Steil GM, Wypij D, Costello JM, Laussen PC, Langer M, et al. Tight glycemic control versus standard care after pediatric cardiac surgery. $N$ Engl J Med. (2012) 367:1208-19. doi: 10.1056/NEJMoa1206044

4. Egi M, Bellomo R, Reade MC. Is reducing variability of blood glucose the real but hidden target of intensive insulin therapy. Crit Care. (2009) 13:30207. doi: $10.1186 /$ cc7755

5. Egi M, Bellomo R, Stachowski E, French CJ, Hart G. Variability of blood glucose concentration and short-term mortality in critically ill patients. Anesthesiology. (2006) 105:244-52. doi: 10.1097/00000542-200608000-00006 further inquiries can be directed to the corresponding author/s.

\section{ETHICS STATEMENT}

Written informed consent was obtained from the individual(s), and minor(s)' legal guardian/next of kin, for the publication of any potentially identifiable images or data included in this article.

\section{AUTHOR CONTRIBUTIONS}

JL participated in the study design, project management, and supervision. $\mathrm{MD}$ and $\mathrm{WL}$ were responsible for the conceptualization and design of forms, data management and quality control, writing, and editing. JL, BH, YZ, GZ, JJ, and $\mathrm{HF}$ were responsible for the project implementation organization and management and data quality control. MD and YL were responsible for the statistical analysis and documentation. $\mathrm{MD}, \mathrm{WL}, \mathrm{FL}, \mathrm{JC}, \mathrm{LD}, \mathrm{DX}$, and $\mathrm{KY}$ were responsible for the recruitment of participants and documentation of data. All authors were contributed to this article and approved the submitted version.

\section{FUNDING}

This study was supported by the General Project Fund for National Clinical Research Center for Child Health and Disorders (grant number: NCRC-2019-GP-12).

\section{ACKNOWLEDGMENTS}

All authors thank the study participants and the participating institutions and the nursing staff for their support and contributions to the study.

\section{SUPPLEMENTARY MATERIAL}

The Supplementary Material for this article can be found online at: https://www.frontiersin.org/articles/10.3389/fnut.2022. 757982/full\#supplementary-material

6. Lazzeri C, Bonizzoli M, Cianchi G, Ciapetti M, Socci F, Peris A. The prognostic role of peak glycemia and glucose variability in trauma: a single-center investigation. Acta Diabetol. (2020) 57:931-35. doi: 10.1007/s00592-020-01493-w

7. Donati A, Damiani E, Domizi R, Botticelli L, Castagnani R, Gabbanelli V, et al. Glycaemic variability, infections and mortality in a medical-surgical intensive care unit. Crit Care Resusc. (2014) 16:13-23.

8. Krinsley JS. Glycemic variability: a strong independent predictor of mortality in critically ill patients. Crit Care Med. (2008) 36:300813. doi: 10.1097/CCM.0b013e31818b38d2

9. Chao WC, Tseng CH, Wu CL, Shih SJ, Yi CY, Chan MC. Higher glycemic variability within the first day of ICU admission is associated with increased 30-day mortality in ICU patients with sepsis. Ann Intensive Care. (2020) 10:17-27. doi: 10.1186/s13613-020-0635-3

10. Zhang X, Zhang J, Li J, Gao Y, Li R, Jin X, et al. Relationship between 24-h venous blood glucose variation and mortality among patients with acute respiratory failure. Sci Rep. (2021) 11:7747-53. doi: 10.1038/s41598-021-87409-2 
11. Wintergerst KA, Buckingham B, Gandrud L, Wong BJ, Kache S, Wilson DM. Association of hypoglycemia, hyperglycemia, and glucose variability with morbidity and death in the pediatric intensive care unit. Pediatrics. (2006) 118:173-82. doi: 10.1542/peds.2005-1819

12. Rake AJ, Srinivasan V, Nadkarni V, Kaptan R, Newth CJ. Glucose variability and survival in critically ill children: allostasis or harm. Pediatr Crit Care Med. (2010) 11:707-12. doi: 10.1097/PCC.0b013e3181e88b1f

13. Meynaar IA, Eslami S, Abu-Hanna A, van der Voort P, de Lange DW, de Keizer N. Blood glucose amplitude variability as predictor for mortality in surgical and medical intensive care unit patients: a multicenter cohort study. $J$ Crit Care. (2012) 27:119-24. doi: 10.1016/j.jcrc.2011.11.004

14. Chao HY, Liu PH, Lin SC, Chen CK, Chen JC, Chan YL, et al. Association of in-hospital mortality and dysglycemia in septic patients. PLoS ONE. (2017) 12:e0170408. doi: 10.1371/journal.pone.0170408

15. Ali NA, O’Brien JM Jr, Dungan K, Phillips G, Marsh CB, Lemeshow S, et al. glucose variability and mortality in patients with sepsis. Crit Care Med. (2008) 36:2316-21. doi: 10.1097/CCM.0b013e3181810378

16. Hermanides J, Vriesendorp TM, Bosman RJ, Zandstra DF, Hoekstra JB, Devries JH. Glucose variability is associated with intensive care unit mortality. Crit Care Med. (2010) 38:838-42. doi: 10.1097/CCM.0b013e3181cc4be9

17. Du YH, Liu CJ, Li J, Dang HX, Zhou F, Sun YL, et al. Glycemic Variability: an independent predictor of mortality and the impact of age in pediatric intensive care unit. Front Pediatr. (2020) 8:403-11. doi: 10.3389/fped.2020.00403

18. Brodovicz KG, Mehta V, Zhang Q, Zhao C, Davies MJ, Chen J, et al. Association between hypoglycemia and inpatient mortality and length of hospital stay in hospitalized, insulin-treated patients. Curr Med Res Opin. (2013) 29:101-07. doi: 10.1185/03007995.2012.754744

19. Srinivasan V, Spinella PC, Drott HR, Roth CL, Helfaer MA, Nadkarni V. Association of timing, duration, and intensity of hyperglycemia with intensive care unit mortality in critically ill children. Pediatr Crit Care Med. (2004) 5:329-36. doi: 10.1097/01.PCC.0000128607.68261.7C

20. Elkon B, Cambrin JR, Hirshberg E, Bratton SL. Hyperglycemia: an independent risk factor for poor outcome in children with traumatic brain injury*. Pediatr Crit Care Med. (2014) 15:623-31. doi: 10.1097/PCC.0000000000000170

21. Bhutia TD, Lodha R, Kabra SK. Abnormalities in glucose homeostasis in critically ill children. Pediatr Crit Care Med. (2013) 14:e16-25. doi: 10.1097/PCC.0b013e3182604998

22. Hirshberg E, Larsen G, Van Duker H. Alterations in glucose homeostasis in the pediatric intensive care unit: hyperglycemia and glucose variability are associated with increased mortality and morbidity. Pediatr Crit Care Med. (2008) 9:361-66. doi: 10.1097/PCC.0b013e318172d401

23. Slater A, Shann F, Pearson G. Pediatric Index of Mortality (PIM) Study Group: PIM2: a revised version of the Pediatric Index of Mortality. Intensive Care Med. (2003) 29:278-85. doi: 10.1007/s00134-002-1601-2

24. Sadan O, Feng C, Vidakovic B, Mei Y, Martin K, Samuels O, et al. glucose variability as measured by inter-measurement percentage change is predictive of in-patient mortality in aneurysmal subarachnoid hemorrhage. Neurocrit Care. (2020) 33:458-67. doi: 10.1007/s12028-019-00906-1

25. Agus MS, Wypij D, Hirshberg EL, Srinivasan V, Faustino EV, Luckett PM, et al. Tight glycemic control in critically ill children. N Engl J Med. (2017) 376:729-41. doi: 10.1056/NEJMoa1612348

26. Nayak PP, Davies P, Narendran P, Laker S, Gao F, Gough SC, et al. Early change in blood glucose concentration is an indicator of mortality in critically ill children. Intensive Care Med. (2013) 39:123-28. doi: 10.1007/s00134-012-2738-2

27. Rhodes A, Evans LE, Alhazzani W, Levy MM, Antonelli M, Ferrer R, et al. Surviving sepsis campaign: international guidelines for management of sepsis and septic shock: 2016. Intensive Care Med. (2017) 43:30477. doi: 10.1007/s00134-017-4683-6

28. Hokka M, Egi M, Mizobuchi S. Glycated hemoglobin Alc level on the day of emergency surgery is a marker of premorbid glycemic control: a retrospective observational study. BMC Anesthesiol. (2018) 18:18086. doi: 10.1186/s12871-018-0641-2

29. Pencina MJ, Sr DRB, D'Agostino RB Jr, Vasan RS. Evaluating the added predictive ability of a new marker: from area under the ROC curve to reclassification and beyond. Stat Med. (2008) 27:157-72. doi: 10.1002/sim.2929
30. Bajaj NS, Osborne MT, Gupta A, Tavakkoli A, Bravo PE, Vita T, et al. Coronary microvascular dysfunction and cardiovascular risk in obese patients. J Am Coll Cardiol. (2018) 72:707-17. doi: 10.1016/j.jacc.2018.05.049

31. Doola R, Greer RM, Hurford R, Flatley C, Forbes JM, Todd AS, et al. Glycaemic variability and its association with enteral and parenteral nutrition in critically ill ventilated patients. Clin Nutr. (2019) 38:170712. doi: 10.1016/j.clnu.2018.08.001

32. Pinchefsky EF, Hahn CD, Kamino D, Chau V, Brant R, Moore AM, et al. Hyperglycemia and glucose variability are associated with worse brain function and seizures in neonatal encephalopathy: a prospective cohort study. J Pediatr. (2019) 209:23-32. doi: 10.1016/j.jpeds.2019.02.027

33. Yu SH, Yu JM, Yoo HJ, Lee SJ, Kang DH, Cho YJ, et al. anti-proliferative effects of rutin on OLETF rat vascular smooth muscle cells stimulated by glucose variability. Yonsei Med J. (2016) 57:373-81. doi: 10.3349/ymj.2016.57.2.373

34. Zhang ZY, Miao LF, Qian LL, Wang N, Qi MM, Zhang YM, et al. Molecular mechanisms of glucose fluctuations on diabetic complications. Front Endocrinol (Lausanne). (2019) 10:640-51. doi: 10.3389/fendo.2019.00640

35. Hu Z, Fang W, Liu Y, Liang H, Chen W, Wang H. Acute glucose fluctuation promotes RAGE expression via reactive oxygen speciesmediated NF-кB activation in rat podocytes. Mol Med Rep. (2021) 23:33039. doi: 10.3892/mmr.2021.11969

36. Bagshaw SM, Bellomo R, Jacka MJ, Egi M, Hart GK, George C. The impact of early hypoglycemia and blood glucose variability on outcome in critical illness. Crit Care. (2009) 13:R91. doi: 10.1186/cc7921

37. Quagliaro L, Piconi L, Assaloni R, Martinelli L, Motz E, Ceriello A. Intermittent high glucose enhances apoptosis related to oxidative stress in human umbilical vein endothelial cells: the role of protein kinase C and NAD(P)H-oxidase activation. Diabetes. (2003) 52:2795804. doi: 10.2337/diabetes.52.11.2795

38. Maeda M, Hayashi T, Mizuno N, Hattori Y, Kuzuya M. Intermittent high glucose implements stress-induced senescence in human vascular endothelial cells: role of superoxide production by NADPH oxidase. PLoS ONE. (2015) 10:e0123169. doi: 10.1371/journal.pone.01 23169

39. La Sala L, Pujadas G, De Nigris V, Canivell S, Novials A, Genovese S, et al. Oscillating glucose and constant high glucose induce endoglin expression in endothelial cells: the role of oxidative stress. Acta Diabetol. (2015) 52:50512. doi: 10.1007/s00592-014-0670-3

40. Jacobi J, Bircher N, Krinsley J, Agus M, Braithwaite SS, Deutschman C, et al. Guidelines for the use of an insulin infusion for the management of hyperglycemia in critically ill patients. Crit Care Med. (2012) 40:325176. doi: 10.1097/CCM.0b013e3182653269

41. Weiss SL, Peters MJ, Alhazzani W, Agus MSD, Flori HR, Inwald DP, et al. Surviving sepsis campaign international guidelines for the management of septic shock and sepsis-associated organ dysfunction in children. Intensive Care Med. (2020) 46:10-67. doi: 10.1007/s00134-019-05878-6

42. Krinsley JS, Preiser JC. Time in blood glucose range 70 to $140 \mathrm{mg} / \mathrm{dl}>80 \%$ is strongly associated with increased survival in non-diabetic critically ill adults. Crit Care. (2015) 19:179-90. doi: 10.1186/s13054-015-0908-7

Conflict of Interest: The authors declare that the research was conducted in the absence of any commercial or financial relationships that could be construed as a potential conflict of interest.

Publisher's Note: All claims expressed in this article are solely those of the authors and do not necessarily represent those of their affiliated organizations, or those of the publisher, the editors and the reviewers. Any product that may be evaluated in this article, or claim that may be made by its manufacturer, is not guaranteed or endorsed by the publisher.

Copyright (C) 2022 Dong, Liu, Luo, Li, Huang, Zou, Liu, Zhang, Chen, Jiang, Duan, Xiong, Fu and Yu. This is an open-access article distributed under the terms of the Creative Commons Attribution License (CC BY). The use, distribution or reproduction in other forums is permitted, provided the original author $(s)$ and the copyright owner(s) are credited and that the original publication in this journal is cited, in accordance with accepted academic practice. No use, distribution or reproduction is permitted which does not comply with these terms. 